\title{
Thyroid hormone regulation of apoptotic tissue remodeling during anuran metamorphosis
}

\author{
SHI Yun-Bo*, Liezhen FU, Shao Chung Victor HSIA, Akihiro TOMitA, Daniel BUCHHOLZ \\ Laboratory of Gene Regulation and Development, National Institute of Child Health and Human Development, \\ National Institutes of Health, Bethesda, Maryland 20892-5431, USA
}

\begin{abstract}
Anuran metamorphosis involves systematic transformations of individual organs in a thyroid hormone (TH)-dependent manner. Morphological and cellular studies have shown that the removal of larval organs/ tissues such the tail and the tadpole intestinal epithelium is through programmed cell death or apoptosis. Recent molecular investigations suggest that $\mathrm{TH}$ regulates metamorphosis by regulating target gene expression through thyroid hormone receptors (TRs), which are DNA-binding transcription factors. Cloning and characterization of $\mathrm{TH}$ response genes show that diverse groups of early response genes are induced by $\mathrm{TH}$. The products of these $\mathrm{TH}$ response genes are believed to directly or indirectly affect the expression and/or functions of cell death genes, which are conserved at both sequence and function levels in different animal species. A major challenge for future research lies at determining the signaling pathways leading to the activation of apoptotic processes and whether different death genes are involved in the regulation of apoptosis in different tissues/organs to effect tissue-specific transformations.
\end{abstract}

Key words: Xenopus laevis, thyroid hormone receptor, extracellular matrix, apoptosis.

\section{INTRODUCTION}

Anuran metamorphosis is a postembryonic developmental process initiated and controlled by thyroid hormone (TH)[1],[2]. TH exerts its effects on target tissues via binding to thyroid hormone receptors (TRs), which are transcription factors that belong to the nuclear receptor super-family. TR modulates gene expression by binding to specific DNA sequences in target genes, most likely by forming a heterodimer with retinoid X receptors (RXRs, or 9cis retinoic acid receptors)[3],[4]. Thus, it is believed that $\mathrm{TH}$ induces a gene regulation cascade in indi-

\footnotetext{
* Corresponding Author: Dr.Yun-Bo Shi, (301) 402-1004(Tel), (301) 402-1323(fax), shi@helix.nih.gov

Abbreviations: TH, Thyroid Hormone; TR, Thyroid Hormone Receptor; MMP, Matrix metalloproteinase; ECM, Extracellular matrix.Received Nov-8-2000 Revised Dec-26-2000 Accepted Jan-9-2001
}

vidual tissues/organs to effect their transformations.

Although TH controls all transformations during metamorphosis, individual tissues/organs undergo distinct changes at different developmental stage[1],[2]. Some organs, such as the limbs, develop de novo, likely from undifferentiated cells preserved in the tadpoles. Others, such as the tail, undergo complete resorption. The vast majority of the organs, such as the intestine and brain, are present both in the tadpole and frog. They undergo partial yet drastic remodeling to adapt to their function in the frog, which have different diets and living habitats. Extensive morphological, cytological, and biochemical analyses have shown that apoptosis is an essential aspect of many of the changes during metamorphosis. In this article, we will first review some of these studies. We will then provide a brief summary on 
the genes known to be involved in the regulation and execution of apoptosis based on studies in different animal species. Finally, we will discuss the molecular pathways induced by $\mathrm{TH}$ that lead to apoptotic tissue transformations during metamorphosis.

\section{APOPTOSIS DURING ANURAN METAMORPHOSIS}

Many larval tissues are removed during metamorphosis and it is presumed that their removal is due to cell death caused by $\mathrm{TH}$ on these target tissues (Fig 1). Kerr et al[5] were the first to demonstrate that apoptosis occurs during anuran metamorphosis. They examined electron microscopically the resorption of the tail muscle and epidermal cells during metamorphosis of the dwarf tree frog Litoria glauerti. They found that the two major cell types, epidermal and muscle cells, of the tail undergo a series of well defined, sequential morphological changes of apoptosis, including the condensation of the cytoplasm and the nuclear chromatin and the subsequent formation of the apoptotic bodies. Similar findings have also been reported for Rana japonica[6] and Xenopus laevis[7].

Intestinal remodeling represents the second type of transformations during metamorphosis. The tadpole intestine is a simple tubular structure consisting of predominantly larval epithelial cells surrounded by sparse connective tissue and muscles (Fig 1)[8]. During metamorphosis, the larval epithelium undergoes complete degeneration and is replaced by adult epithelium. Morphological and biochemical analyses indicate that the larval epithelial cells undergo apoptosis and the apoptotic bodies are removed at least in part through phagocytosis by macrophages migrating over from the connective tissue[8].

While it is expected that tissue resorption and remodeling require removal of larval cells, the de novo development of limbs is likely to involve cell death as well (Fig 1). As the limbs undergo morphogenesis, the inter-digital cells have to be selectively removed, although it remains to be shown that their death is apoptotic.

Several studies indicate that TH appears to induce cell death mainly by targeting the dying cells directly in the tail and intestine. First, isolated epidermal cells from the tail of Rana catesbeiana tadpoles can be induced to die in vitro when cultured in the presence of $\mathrm{TH}[9]$, suggesting that the $\mathrm{TH}$ dependent epidermal cell death is cell autonomous,

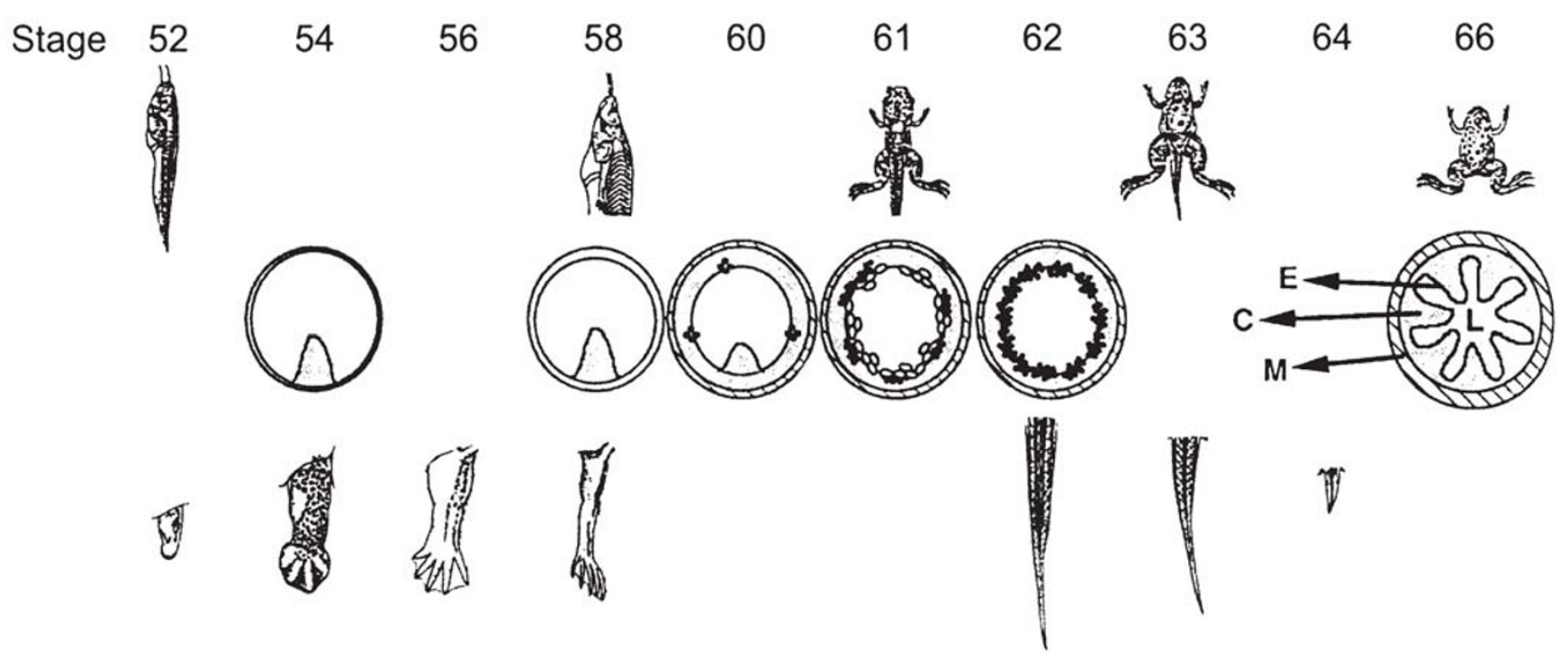

Fig 1. Stage-dependent organ transformations during Xenopus laevis metamorphosis.

The developmental stages are from Nieuwkoop and Faber[54]. The tails at stages 62-66 are drawn to the same scale to show the resorption (no tail remains by stage 66), while the tadpoles, intestinal cross-sections (middle) and the hindlimbs at different stages are not in the same scale in order to show the morphological differences. Tadpole small intestine has a single epithelial fold, where connective tissue (C) is abundant, while a frog has a multiply folded intestinal epithelium (E), with elaborate connective tissue and muscle (M). Dots: proliferating adult intestinal epithelial cells. Open circles: apoptotic primary intestinal epithelial cells. L: intestinal lumen. 
at least when cultured in isolation in vitro. Second, a cell line has been established from Xenopus laevis tail muscles and found to respond to TH by undergoing apoptosis[10]. Finally, we have isolated the larval epithelial cells from the intestine of Xenopus laevis tadpoles and cultured them in vitro in the presence or absence of TH[11],[12]. Our results indicate that the larval epithelial cells are induced to die by physiological concentrations of TH. Biochemical and morphological analyses have shown that the THinduced epithelial cell death has all the characteristics of apoptosis, including the formation of apoptotic bodies and nucleosomal sized nuclear DNA fragments. Interestingly, when the larval intestinal epithelial cells were cultured on plastic dishes coated with various components of the ECM, such as fibronectin and laminin, etc., the $\mathrm{TH}$-induced cell death was inhibited[11]. However, the ECM components failed to influence TH-stimulated cellular DNA synthesis and the downregulation of two epithelial specific genes. These results indicate that the ECM preferentially affects cell death. They further suggest that the apoptosis is not entirely cell autonomous in vivo but depends on cell-cell and/or cellECM interactions. Such a conclusion is also consistent with findings from organ culture studies. For example, the removal of tail epidermis prevents $\mathrm{TH}$ induced tail resorption in organ cultures[13]. On the other hand, there is also evidence to suggest that adult-type non- $T$ leukocytes may participate in the specific elimination of larval tail cells[14]. In addition, when intestinal fragments are cultured in the presence of $\mathrm{TH}$, larval cell death takes place within 2-3 days, followed by the development of adult epithelium after 5-7 days[8]. When isolated epithelium is cultured with TH, only cell death occurs. The development of the adult epithelium requires the presence of the connective tissue as co-culturing the larval epithelium and connective tissue restores adult epithelial development. Thus, cell-cell interactions are likely important for both larval tissue degeneration and adult tissue development.

\section{CELL DEATH GENES}

Many genes involved in cell death have been isolated and characterized. The first genes were identified from genetic studies in the nematode Caenorhaditis elegans[15], [16]. During normal de- velopment of C. Elegans, 131 out of the 1090 somatic cells in the developing animal undergo programmed cell death. While the signals for the death of these 131 cells are still under investigation, analyses of mutants that are defected at various steps of the apoptotic process have led to the cloning of many genes involved at the death execution or later steps. Subsequently, the homologs of some of these genes were found in mammals and shown to have similar functions[17-20]. The best studied among them are those acting at the execution step, where three C. Elegans genes, ced-3, ced-4, and ced-9, are involved. The ced- 3 and ced- 4 are cell death promoters, while ced-9 inhibits cell death. The mammalian homologs of these C. Elegans death genes have been cloned and their gene products have been characterized biochemically. The most extensively studied are the ced-3 homologs, which encode cysteine proteases capable of cleaving after an aspartic acid residue within a substrate[17],[21]. Over 10 such proteases, now referred to as caspases, exist in mammals. Upregulation of these caspases through increased expression and/or activation of the proenzymes leads to the degradation of various cellular substrates and cell death. Gene knockout studies in mice provide direct support for different caspases in apoptosis of different tissues/organs in development[22].

The mammalian homologs of ced-9 are the bcl-2 family members[18], [19],[23],[24]. Interestingly, there are two subfamilies within the bcl-2 superfamily, the bcl-2 and bax subfamilies. The bcl2 subfamily members protect cells from apoptosis while the bax subfamily members promote cell death. Many members of this family can form homodimers and/or heterodimers and thus the balance of the two subfamilies can affect cell fate. The exact mechanisms by which they regulate apoptosis remain to be determined. One likely mechanism for the bcl-2 subfamily to inhibit apoptosis was suggested by the cloning and characterization of ced-4 and its mammalian homolog Apaf-1[20],[25]. Apaf-1 (ced-4) can interact directly with caspases (ced-3), thus participating directly in caspase activation. Interestingly, ced-4 also interacts with ced-9 directly, suggesting that ced-9 (bcl-2) may directly inhibit caspase activation through this interaction. In mammals, in addition to Apaf-1, at least another factor, Apaf-3 or cytochrome $\mathrm{C}$, is also required for caspase activation 
[25]. Cytochrome $\mathrm{C}$ resides in the space between the inner and outer membranes of mitochondria. There is evidence to suggest that bcl-2 family members can regulate caspases activation by influencing cytochrome $\mathrm{C}$ release either directly or indirectly.

There are at least two other classes of proteins participating in apoptotic execution or its regulation in mammals. The first are the inhibitors of apoptosis (IAPs)[25],[26]. These proteins bind to caspases directly and thus block caspase function. Their degradation appears to be an important factor in the commitment of cells to apoptosis. The second class includes nucleases that degrade nuclear DNA during apoptosis[20],[27-29]. The best characterized is the DNA fragmentation factor (DFF), which is a heterodimer of caspase-activated DNase (CAD) or DFF40 and inhibitor of CAD (ICAD) or DFF45[27]. While CAD can cleave chromosomal DNA to generate the nucleosomal DNA ladder that is commonly observed in vertebrate apoptosis, the heterodimer is inactive. The cleavage of ICAD triggered by apoptotic signals activates $\mathrm{CAD}$ and leads to the cleavage of nuclear DNA, thus irreversibly committing the cell to the apoptotic pathway.

In addition to the above genes, there are also many genes involved in the subsequent steps, i.e., the engulfment and degradation of dead cells or the resulting apoptotic bodies[30], [31]. Relatively little is known about these steps in mammals. On the other hand, it is expected that like the other genes partici- pating in execution of apoptosis as described above, these genes are likely to be conserved among different apoptotic processes.

\section{GENE REGULATION BY TR $_{\text {}}$}

$\mathrm{TH}$ is believed to induce a series of gene regulation steps during metamorphosis. The first step is the transcriptional regulation of direct TH response genes by TRs (Fig 2). TRs are transcription factors that recognize specific DNA sequences, or TH response elements (TREs), in their target genes. $\mathrm{Nu}-$ merous in vitro and tissue culture cells studies as well as in vivo functional studies in developing Xenopus laevis indicate that TRs are constitutively bound to TREs in chromatin as heterodimers formed with RXRs[3, 4, 32-34]. In the absence of TH, TR/ RXR heterodimers can recruit corepressor complexes containing histone deacetylases to the target genes, which leads to histone deacetylation to facilitate gene repression by the receptors. When TH is present, the corepressor complexes are released and are replaced by coactivator complexes, which often have histone acetyl transferase activity. Thus, it is hypothesized that $\mathrm{TH}$ activates gene transcription in part through increasing local histone acetylation (Fig 2).

Our own studies in the reconstituted TH-dependent Xenopus oocyte system and our analyses on endogenous TH-response genes during Xenopus development have shown directly that TR/RXR heterodimers are bound to TRE in chromatin in in
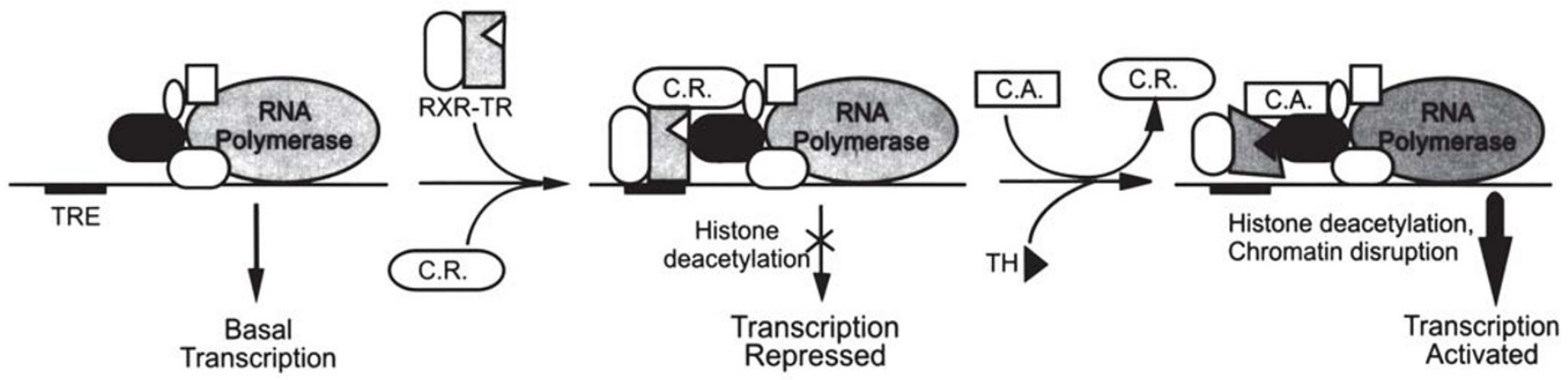

Fig 2. A model for transcriptional regulation by TR. TR is assumed to form a heterodimer with RXR. The heterodimer can bind to the TRE in a target gene. The binding of an unliganded TR-RXR heterodimer leads to transcriptional repression possibly through the recruitment of a corepressor complex (C.R.) and/or interaction with the basal transcription machinery. Upon binding by TH, a conformational change takes place in the heterodimer, which may be responsible for the release of the corepressor and recruitment of a coactivator complex (C.A.), thus leading to transcriptional activation. In addition to RNA polymerase, many other factors are required as depicted in part in the Figure. 
vivo and repress target gene expression in a process requiring histone deacetylase activity[32],[33]. Furthermore, TH treatment of premetamorphic tadpoles leads to local increase in histone acetylation specifically at the target genes at least in the tail and the intestine, both of which undergo extensive apoptotic degeneration of the larval tissues. In addition, treating the tadpoles with the histone deacetylase inhibitor trichostatin A can activate the $\mathrm{TH}$ responses genes and increase histone acetylation levels at TH target genes in these organs. Thus, unliganed TRs appear to recruit histone deacetylase complexes to repress gene expression at least in these two organs. In support of this, a known TR-binding corepressor, $\mathrm{N}$-CoR, is expressed in premetamorphic tadpoles (Sachs and Shi, unpublished data) and at least two N-CoR containing histone deacetylase complexes exist in Xenopus[35]. The release of these or other corepressor complexes upon $\mathrm{TH}$ binding to TRs and the concurrent recruitment of coactivator complexes, which remain to be characterized in amphibians, are likely responsible for the activation of genes involved in metamorphosis. The presence of different corepressor or coactivator complexes and variation in the levels of such complexes may play an important role in determining the gene regulation programs induced by $\mathrm{TH}$ in individual organs/ tissues.

\section{GENES INVOLVED IN TH-INDUCED APOPTOSIS}

\section{Early $T H$ response genes}

The dependence of anuran metamorphosis on $\mathrm{TH}$ allows one to identify genes that are involved in mediating the effects of the TH signal in apoptosis in different tadpole tissues by using various molecular approaches. Many of the genes regulated by TH during Xenopus laevis metamorphosis have been isolated by using PCR-based subtractive hybridization with mRNAs from different organs of premetamorphic tadpoles with or without TH treatment[2]. Characterization of the early genes, i.e., those regulated by $\mathrm{TH}$ within a $24 \mathrm{~h}$ treatment period, shows that diverse groups of genes are induced by $\mathrm{TH}$ in organs such as the limb, tail, intestine, and brain. In general, these $\mathrm{TH}$ response genes include transcription factors, signaling molecules, matrix metalloproteinases (MMPs), and others.
Most of the transcription factors and MMPs are expressed in different organs and their spatial and temporal regulation during development and $\mathrm{TH}$ treatment suggests that they act upstream of the gene regulation steps that specify tissue specific changes during metamorphosis. The transcription factors are expected to regulate downstream genes directly. Currently, little is known about their target genes and their roles in larval cell death remains to be established.

We have been studying the roles of MMPs in larval tissue degeneration. MMPs are $\mathrm{Zn}^{2+}$-dependent proteinases that are extracellular or bound to plasma membrane[36-40]. They can cleave specific proteinaceous components of the extracelluar matrix (ECM) and non-ECM proteins. Numerous studies in mammals have suggested that MMPs are involved in diverse developmental and pathological processes by modifying or degrading the ECM, thus influencing cell behavior[41-43]. Through gene expression analyses, we and others have shown that Xenopus collagenase-3, collagenase- 4 , and stromelysin-3 (ST3) are expressed in tissues where apoptosis occurs during metamorphosis[33]. In particular, we have demonstrated a tight spatial and temporal correlation of ST3 mRNA and protein with ECM remodeling and epithelial apoptosis during intestinal metamorphosis(Fig 3). Organ culture studies have provided direct support for a requirement of MMP activity in TH-induced epithelial cell death in the intestine (Fig 4)[45]. Thus, ST3 may modify the ECM to influence larval cell death. Further studies are needed to determine the signaling pathways mediating the effect of ST3.

\section{Late $\mathrm{TH}$ response genes}

Noticeably absent in the original screens for early $\mathrm{TH}$ response genes are cell death genes described above. This may not be surprising considering that cell death is a late event and that cell death genes are unlikely regulated by $\mathrm{TH}$ early in the gene regulation cascade. Interestingly, a PCR-based subtractive screen for genes regulated in the Xenopus intestine after a 4-day $\mathrm{TH}$ treatment of premetamorphic tadpoles also failed to isolate any cell death genes[2],[46]. It did, however, isolated many late $\mathrm{TH}$ response genes. These genes again fall into several categories, including transcription factors and signaling molecules. The isolation of such genes is 
consistent with the idea that multiple gene regulation steps are needed to complete the transformation of the intestine. Unfortunately, few studies have been carried out to reveal any potential roles of these genes in apoptosis.

\section{Cell death genes during metamorphosis}

Although no cell death genes were isolated in various screens for $\mathrm{TH}$ response genes, the similar biochemical and morphological properties between apoptosis during anuran metamorphosis and that in other animal species suggest the involvement of similar cell death genes. This is further supported by the ability of known apoptosis inhibitors, i.e., those against caspases and nucleases that degrade proteins and DNA, respectively, to inhibit TH-induced apoptosis in primary cultures of tadpole intestinal epithelial cells[11] or in cultures of a cell line derived from tadpole tail muscles[10].

Based on the likely conservation in sequences among anuran and mammalian cell death genes, several genes encoding caspases and bcl-2 family members have been cloned in Xenopus laevis[47], [48]. Sequence analyses have shown that these genes are indeed highly homologous to their mammalian counterpart and have similar molecular functions when expressed in tissue culture cells.

The Xenopus caspase genes are expressed in the tail during metamorphosis[10],[47]. While they are upregulated by $\mathrm{TH}$ treatment of premetamorphic tadpoles, their upregulation by $\mathrm{TH}$ is relativeluy small and requires more than one day of $\mathrm{TH}$ treatment. Thus, they are late TH response genes and their small magnitudes of regulation by TH may

\section{ST3 sense ST3 anti-sense TUNEL}

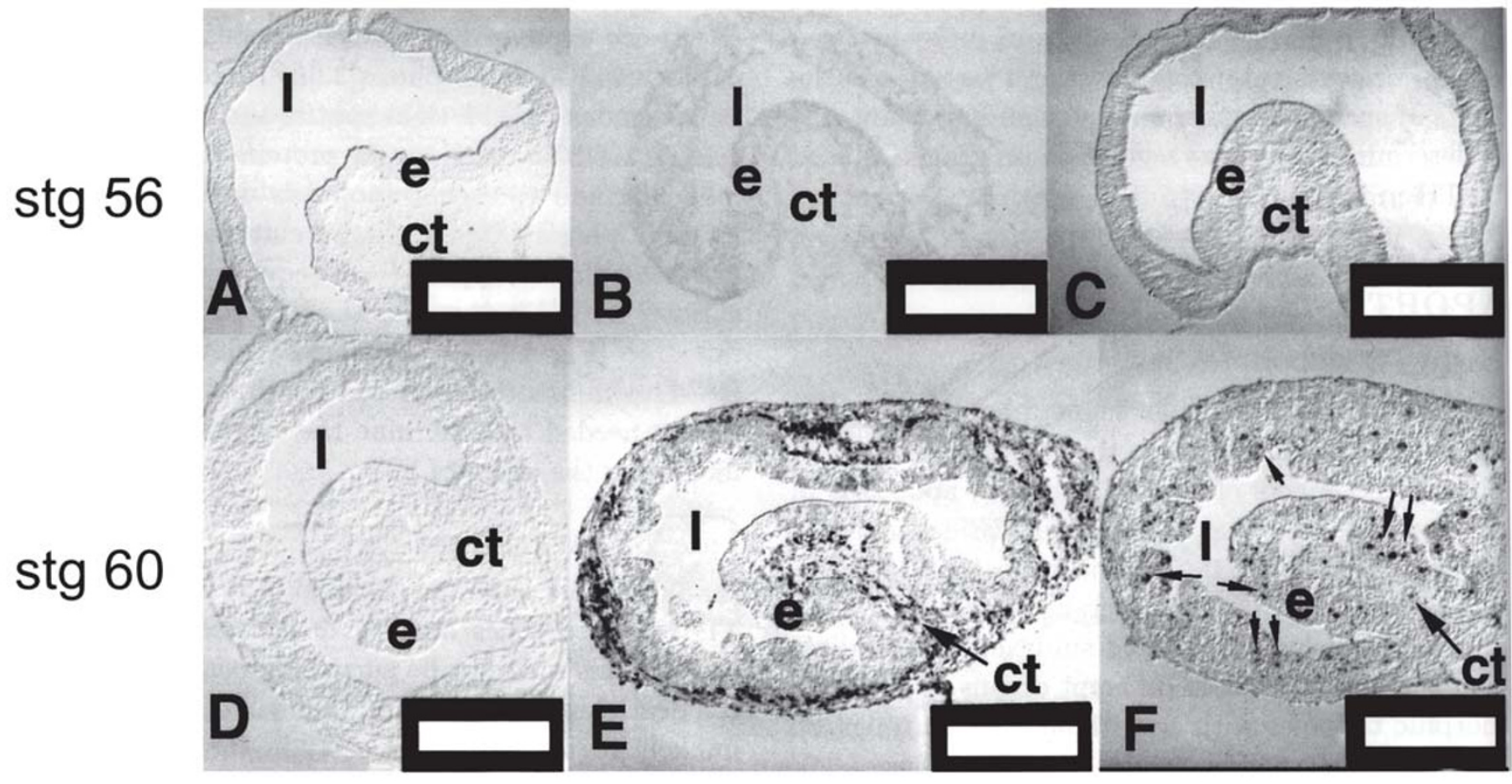

Fig 3.

Association of ST3 expression with larval epithelial cell death during intestinal remodeling. Stages 56 (A, B, C) and stage 60 (C, D, E) intestines were analyzed by in situ hybridization for ST3 expression (A, B, D, E) and by TUNEL assay for apoptotic cell detection (C, F). There was no detectable ST3 expression at stage 56 (B). ST3 expression was strong in all regions of the connective tissue at stage 60 but not in the epithelium or muscles (E). Likewise, at stage 56, there was no apoptotic signals (C), while there were many apoptotic cells, mainly within the epithelium, at stage 60 (arrows). Note that the control hybridization with the sense RNA probe did not detect any signal, as expected. ct, connective tissue; e, epithelium; l, lumen. White bars: 250 $\mu \mathrm{m}$. From Damjanovski et al.[55]. 
explain why they escaped the differential screens. Their presence during metamorphosis suggests that they participate in $\mathrm{TH}$-induced apoptosis.

The two bcl-2 family members cloned in Xenopus belong to the subfamily of cell death inhibitors [48],[49]. They are capably of inhibiting cell death when expressed in tissue culture cells or in Xenopus tadpoles. On the other hand, limited expression studies suggest that they are expressed at only low levels and/or their expression levels do not change during metamorphosis. Thus, it is unclear whether they play any roles in cell fate regulation during metamorphosis.

\section{CONCLUSION AND PROSPECTS}

Programmed cell death or apoptosis plays important roles in development of animals from C. elegans, Drosophila, to human, and is critical for the physiological function of many organs, such as the immune system[50-53]. Anuran metamorphosis is a postembryonic process that is absolutely dependent upon the presence of TH. The degeneration of larval tissues through programmed cell death during this process offers a unique opportunity to study the molecular pathways that lead to apoptosis of different cell types during postembryonic development in vertebrates. Existing studies suggest that the apoptosis during anuran metamorphosis likely utilize the same cell death genes as those in mammals. The cloning and functional characterization of the cell death genes should provide insights on whether different cell death genes are involved in the apoptosis of different larval tissues. The causative

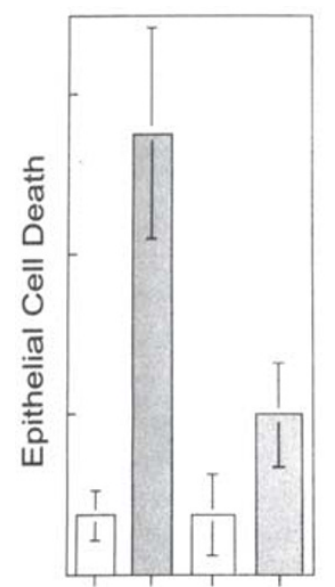

$-\mathrm{TH}+\mathrm{TH}-\mathrm{TH}+\mathrm{TH}$ -inh. -inh. +inh.+inh.
Fig 4.

Blocking MMP function inhibits TH-induced epithelial apoptosis. Intestinal explants were cultured for 3 days in the presence or absence of TH and/ or a synthetic MMP inhibitor (inh.). The apoptosis in the epithelium was detected with the TUNEL method and the number of labeled nuclei (apoptotic cells) was quantified for each treatment. See Ishizuya-Oka et al.[45] for more details. effects of TH on metamorphosis are mediated by TRs. The analyses of the TH response genes suggest that $\mathrm{TH}$ induces initially the expression of several diverse groups of genes. A major challenge will be to determine how these early genes in turn influence the expression and/or the function of cell death genes. The availability of a large array of molecular and genetic approaches should make such endeavors not only feasible but also highly rewarding in the years to come.

\section{REFERENCES}

[1] Dodd MHI, Dodd JM. The biology of metamorphosis. In: Lofts B (ed.) Physiology of the amphibia. Acadmic Press, New York 1976:467-599.

[2] Shi Y-B. Amphibian Metamorphosis: From morphology to molecular biology. John Wiley and Sons, Inc., New York 1999.

[3] Tsai MJ, O'Malley BW. Molecular mechanisms of action of steroid/thyroid receptor superfamily members. Ann Rev Biochem 1994; 63:451-86.

[4] Mangelsdorf DJ et al. The nuclear receptor superfamily: the second decade. Cell 1995; 83:835-9.

[5] Kerr JFR et al. An electron-microscope study of cell eletion in the anuran tadpole tail during spontaneous metamorphosis with special reference to apoptosis of striated muscle fibres. J Cell Sci 1974; 14:571-85.

[6] Kinoshita T et al. Autolysis and heterolysis of the epidermal cells in anuran tadpole tail regression. J of Morphology $1985 ; \mathbf{1 8 5}: 269-75$.

[7] Nishikawa A, Hayashi H. Spatial, temporal and hormonal regulation of programmed muscle cell death during metamorphosis of the frog Xenopus laevis. Differentiation 1995; 59:207-14.

[8] Shi Y-B, Ishizuya-Oka A. Biphasic intestinal development in amphibians: Embryogensis and remodeling during metamorphosis. Current Topics in Develop Biol 1996; 32: 205-35.

[9] Nishikawa A, Yoshizato K. Hormonal regulation of growth and life span of bullfrog tadpole tail epidermal cells cultured in vitro. J Exp Zool 1986; 237:221-30.

[10] Yaoita Y, Nakajima K. Inductoin of Apoptosis and CPP32 Expression by Thyroid Hormone in a Myoblastic Cell Line Dervided from Tadpole Tail. J Biol Chem 1997; 272:51227.

[11] Su Y et al. Thyroid hormone induces apoptosis in primary cell cultures of tadpole intestine: cell type specificity and effects of extracellular matrix. J Cell Biol 1997; 139: 1533-43.

[12] Su Y et al. Cyclosporin A But not FK506 Inhibits Thyroid Hormone-Induced Apoptosis in Xenopus Tadpole Intestinal Epithelium. FASEB J 1997; 11:559-65.

[13] Niki K et al. Epidermal tissue requirement for tadpole tail regression induced by thyroid hormone. Dev Biol 1982; 94:116-20.

[14] Izutsu Y et al. Adult-type splenocytes of Xenopus induce apoptosis of histocompatible larval tail cells in vitro. 
Differentiation 1996; 60:277-86.

[15] Ellis HM, Horvitz HR. Genetic control of programmed cell death in the nematode C. elegans. Cell 1986; 44:81729.

[16] Hengartner MO, Horvitz HR. The ins and outs of programmed cell death during C. elegans development. Philos Trans R Soc Lond B Biol Sci 1994; 345:243-6.

[17] Cryns V, Yuan J. Proteases to die for [published erratum appears in Genes Dev 1999 Feb 1; 13(3):371. Genes Dev 1998; 12:1551-70.

[18] Rao L, White E. Bcl-2 and the ICE family of apoptotic regulators: making a connection. Curr Opin Genet Dev 1997; 7:52-8.

[19] Adams JM, Cory S. The Bcl-2 protein family: arbiters of cell survival. Science 1998; 281:1322-6.

[20] Shi Y-B et al. Regulation of apoptosis during development: Input from the extracellular matrix. Intern $\mathrm{J}$ of $\mathrm{Mol}$ Medicine 1998; 2:273-82.

[21] Alnemri ES et al. Human ICE/CED-3 protease nomenclature [letter]. Cell 1996; 87:171.

[22] Woo M et al. Executionary pathway for apoptosis: lessons from mutant mice. Cell Research 2000; 10:267-78.

[23] Yin X-M. Signal transduction mediated by Bid, a prodeath Bcl-2 family proteins, connects the death receptor and mitochondria apoptosis pathways. Cell Res 2000; 10: 161-7.

[24] Gross A et al. BCL-2 family members and the mitochondria in apoptosis. Genes and Dev 1999; 13:1899-911.

[25] Budihardjo I et al. Biochemical pathways of caspase activation during apoptosis. Annu Rev Cell Dev Biol 1999; 15:269-90.

[26] Yang Y L, M. L X. The IAP family: endogenous caspase inhibitors with multiple biological activities. Cell Research 2000; 10:169-77.

[27] Zhang J H, Xu M. DNA fragmentation in apoptosis. Cell Research 2000; 10:205-11.

[28] Wu Y-C et al. NUC-1 a Caenorhabditis elegans DNase II homolog, functions in an intermediate step of DNA degardation during apoptosis. Genes and Dev 2000; 14: 536-48.

[29] Li L Y et al. Endonuclease G is an apoptotic DNase when released from mitochondria. Nature 2001; 412:95-9.

[30] Platt N et al. Recognizing death: the phagocytosis of apoptotic cells. Trends Cell Biol 1998; 8:365-72.

[31] Savill J. Apoptosis. Phagocytic docking without shocking [news; comment]. Nature 1998; 392:442-3.

[32]Sachs LM, Shi Y-B. Targeted chromatin binding and histone acetylation in vivo by thyroid hormone receptor during amphibian development. PNAS 2000; 97:1313843.

[33] Shi Y-B, Ishizuya-Oka A. Thyroid hormone regulation of apoptotic tissue remodeling: Implications from molecular analysis of amphibian metamorphosis. Progress in Nucleic Acid Research and Molecular Biology 2001; 65: 53-100.

[34] Yen PM. Physiological and molecular basis of thyroid hormone action. Physiol Rev 2001; 81:1097-142.
[35] Jones PL et al. Multiple N-CoR complexes contain distinct histone deacetylases. J Biol Chem 2001; 276:880711.

[36] Nagase H. Cell surface activation of progelatinase A (proMMP-2) and cell migration. Cell Res 1998; 8:179-86.

[37] Pei D. Leukolysin/MMP25/MT6-MMP: a novel matrix metalloproteinase specifically expressed in the leukocyte lineage. Cell Res 1999; 9:291-303.

[38] Alexander CM, Werb Z. Extracellular matrix degradation. 2nd ed. Plenum Press, New York. 1991.

[39] Birkedal-Hansen $\mathrm{H}$ et al. Matrix metalloproteinases: a review. Crit Rev in Oral Biol and Med 1993; 4:197-250.

[40] McCawley LJ, Matrisian LM. Matrix metalloproteinases: they're not just for matrix anymore! Current Opinion in Cell Biology 2001; 13:534-40.

[41] Sang QX. Complex role of matrix metalloproteinases in angiogenesis. Cell Res 1998; 8:171-7.

[42] Stetler-Stevenson WG. Dynamics of matrix turnover during pathologic remodeling of the extracellular matrix. American Journal of Pathology 1996; 148:1345-50.

[43] Murphy G, Gavrilovic J. Proteolysis and cell migration: creating a path? Current Opinion in Cell Biology 1999; 11:614-21.

[44] Vo TH, Werb Z. Matrix metalloproteinases: effectors of development and normal physiology. Genes and Dev 2000; 14:2123-33.

[45] Ishizuya-Oka A et al. Requirement for matrix metalloproteinase stromelysin-3 in cell migration and apoptosis during tissue remodeling in Xenopus laevis. J Cell Biol 2000; 150:1177-88.

[46] Amano T, Yoshizato K. Isolation of genes involved in intestinal remodeling during anuran metamorphosis. Wound Repair Regen 1998; 6:302-313.

[47] Nakajima K et al. Structure, expression, and function of the Xenopus laevis caspase family. J Biol Chem 2000; 275:10484-91.

[48] Cruz-Reyes J, Tata JR. Cloning, characterization and expresion of two Xenopus bcl-2-like cell-survivial genes. Gene 1995; 158:171-9.

[49] Coen L et al. Xenopus Bcl-XL selectively protects RohonBeard neurons from metamorphic degeneration. Proc Natl Acad Sci USA 2001; 98:7869-674.

[50] Rothstein TL. Inducible resistance to Fas-mediated apoptosis in B cells. Cell Research 2000; 10:245-66.

[51] Lee C-Y, Baehrecke EH. Genetic regulation of programmed cell death in Drosophila. Cell Research 2000; 10:193204.

[52] Donjerkovi'c D, Scott DW. Activation-induced cell death in B lymphocytes. Cell Research 2000; 10:179-92.

[54] Meier PJ et al. Apoptosis in development. Nature 2000; 407:796-801.

[55] Nieuwkoop PD, Faber J. Normal table of Xenopus laevis. North Holland Publishing, Amsterdam 1956.

[56] Damjanovski S et al. Spatial and temporal regulation of collagenases- $3,-4$, and stromelysin -3 implicates distinct functions in apoptosis and tissue remodeling during frog metamorphosis. Cell Research 1999; 9:91-105. 